\title{
Fonctions de la protéine prion PrPc
}

\section{Sylvie Cazaubon, Pedro Viegas, Pierre-Olivier Couraud}

$>$ Les maladies à prion (scrapie du mouton, maladie de la vache folle, maladie de CreutzfeldtJakob chez l'homme) impliquent les deux isoformes, normale $\mathrm{PrP}^{\mathrm{c}}$ et pathologique ou scrapie $\mathrm{PrP}^{\mathrm{sc}}$, de la protéine prion. La protéine $\mathrm{PrP}^{\mathrm{c}}$ est fortement exprimée dans le système nerveux central (neurones, cellules gliales) mais est aussi présente dans les cellules du système immunitaire, et les cellules épithéliales et endothéliales. Des études récentes permettent de classer les fonctions de la $\operatorname{PrP}^{c}$ en deux grands domaines: (1) la survie cellulaire et (2) l'adhérence cellulaire, celle-ci étant liée à différentes fonctions selon le type cellulaire (neuritogenèse, intégrité des barrières épithéliale et endothéliale, migration transendothéliale de monocytes, activation de lymphocytes T). Ces observations suggèrent des fonctions de la PrPc importantes notamment en situations de stress cellulaire inflammatoire et d'infection. <

Après une vingtaine d'années de recherches sur le prion, il est généralement bien établi que la protéine prion PrP est le principal agent causal du développement et de la transmission des maladies à prion, telles que l'encéphalopathie spongiforme bovine et la maladie de Creutzfeldt-Jakob chez l'homme. Ces recherches ont provoqué une véritable révolution scientifique avec l'émergence de deux nouveaux concepts : l'agent pathogène pourrait être une protéine (hypothèse de la protéine seule proposée par Prusiner en 1982, [1]), et la structure tertiaire de cette protéine serait capable de transmettre de l'information et de provoquer l'expression d'un nouveau phénotype. Les deux isoformes conformationnelles de la protéine prion, $\operatorname{Pr} P^{c}$ (isoforme cellulaire normale) et $\operatorname{PrP}^{\text {sc }}$ (isoforme «scrapie » pathologique), seraient ainsi associées à des phénotypes distincts. Cependant, si la neurodégénérescence spongiforme caractéristique des maladies à prion est généralement associée à la présence de $\operatorname{PrPsc}$, les fonctions biologiques de l'isoforme normale $\mathrm{PrP}^{\mathrm{C}}$ sont encore mal connues.

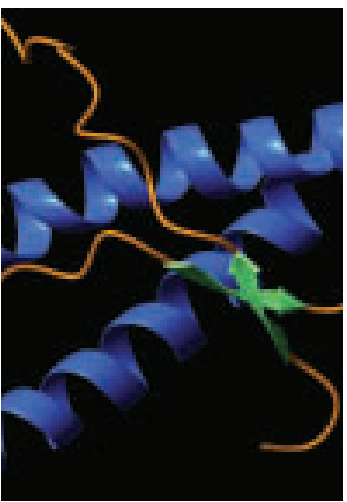

Institut Cochin, Université Paris Descartes, CNRS (UMR 8104), Paris, France. Inserm, U567, Paris, France. Institut Cochin, Département Biologie Cellulaire, 22, rue Méchain, 75014 Paris, France. cazaubon@cochin.inserm.fr

La $\operatorname{PrP}^{c}$ est présente à un niveau élevé dans le cerveau, à la surface des neurones et des cellules gliales, mais elle est également exprimée en périphérie, notamment par les cellules du système immunitaire et les cellules épithéliales et endothéliales constituant les barrières physiologiques de l'organisme: barrière intestinale et barrière vasculaire, respectivement. L'identification des fonctions normales de la $\operatorname{Pr} P^{c}$ à la surface des neurones et de ces autres types cellulaires s'avère donc être fondamentale non seulement pour la compréhension de la progression des maladies à prion, mais aussi pour l'identification des mécanismes moléculaires de régulation physiologique de ces cellules impliquant la $\mathrm{PrP}^{c}$.

\section{Rôle de la PrPc dans la survie cellulaire}

\section{Protection contre le stress oxydant}

Une des premières propriétés de $\operatorname{la} \operatorname{Pr}^{c}$ qui a été mise en évidence est sa capacité de lier le cuivre sous forme d'ion cuivrique $\left(\mathrm{Cu}^{2+}\right)$, et plus faiblement le zinc $\left(\mathrm{Zn}^{2+}\right)$ et le manganèse $\left(\mathrm{Mn}^{2+}\right)$, suggérant une contribution de la $\operatorname{Pr}^{c}$ à la protection contre des concentrations toxiques en ions métalliques [2]. Plusieurs arguments ont été avancés en faveur d'un rôle de la $\operatorname{PrP}^{c}$ dans la protection contre le stress oxydant, probablement par activation de la superoxyde dismutase (SOD) dépendante des ions $\mathrm{Cu}^{2+}$ et $\mathrm{Zn}^{2+}[3]$, et une activité intrinsèque de type SOD a même été proposée pour la $\operatorname{Pr} P^{c}$, mais ces observations restent controversées [3]. Il est intéressant de remarquer qu'une expression augmentée de la $\operatorname{PrP}^{c}$ 
est observée dans d'autres maladies neurodégénératives, maladies d'Alzheimer et de Parkinson, qui sont aussi associées à un stress oxydant impliquant les ions $\mathrm{Cu}^{2+}$ [4]. D'autres pathologies cérébrales (ischémie, gliome) et plusieurs maladies neuromusculaires (dénervation neurogène, myosite à inclusions, polymyosite) s'accompagnent aussi d'une surexpression de la $\operatorname{PrP}^{c}$, ce qui pourrait suggérer son implication dans la réponse physiologique à une situation de stress cellulaire [5]. Inversement, il a été montré que des neurones en culture provenant de souris chez lesquelles le gène codant pour la $\operatorname{PrP}^{c}$ a été invalidé (souris $\operatorname{Pr}^{-/-}$) sont plus sensibles à un stress oxydant induit par des dérivés actifs de l'oxygène [6].

\section{Maintien de l'état d'oxydo-réduction?}

Un couplage fonctionnel de la $\operatorname{Pr}^{c}$ à la NADPH oxydase, conduisant à la production transitoire de dérivés actifs de l'oxygène, a été mis en évidence dans des neurones sérotoninergiques et noradrénergiques [7]. De plus, dans ces cellules, la liaison et l'agrégation de la $\operatorname{Pr}^{c}$ par des anticorps spécifiques induisent l'activation de la voie MAP-kinase $\varepsilon R K$, dépendante de la protéine kinase Fyn [8], dont l'implication dans la survie cellulaire est largement documentée. De ces travaux, on est tenté de conclure que l'activation de ERK par des produits de la NADPH oxydase, observée dans plusieurs types de cellules neuronales et non-neuronales, pourrait constituer une réponse couplée à la $\operatorname{Pr}^{c}$ permettant le maintien de l'état d'oxydo-réduction de la cellule [9].

\section{Type cellulaire
onction anti-apoptotique de la PrP}

\begin{tabular}{|c|c|c|c|}
\hline Type cellulaire & Fonction & $\begin{array}{c}\text { Mécanisme ou } \\
\text { Signalisation intracellulaire }\end{array}$ & Réf \\
\hline \multicolumn{4}{|c|}{ Fonction anti-apoptotique de la PrPc } \\
\hline Neurones & $\begin{array}{l}\text { Protection contre } \\
\text { des concentrations } \\
\text { phamarcologiques de } \mathrm{Cu}^{2+}\end{array}$ & $\begin{array}{l}\text { Liaison du } \mathrm{Cu}^{2+} \\
\text { Internalisation de } \mathrm{PrP}^{\mathrm{c}}-\mathrm{Cu}^{2+}\end{array}$ & [2] \\
\hline Neurones & $\begin{array}{l}\text { Protection contre le stress } \\
\text { oxydant }\end{array}$ & $\begin{array}{l}\text { Activation de la } \mathrm{Cu} / \mathrm{Zn}-\mathrm{SOD} \\
\text { Activité intrinsèque «SOD-like»? }\end{array}$ & [3] \\
\hline $\begin{array}{l}\text { Neurones } \\
\text { (sérotoninergiques, } \\
\text { noradrénergiques) }\end{array}$ & $\begin{array}{l}\text { Survie cellulaire } \\
\text { Prolifération }\end{array}$ & $\begin{array}{l}\text { Couplage à la NADPH oxydase? } \\
\text { Activation de la voie ERK }\end{array}$ & {$[7,9]$} \\
\hline Neurones & $\begin{array}{l}\text { Protection contre l'apoptose } \\
\text { dépendante de } \mathrm{Bax} / \mathrm{Bcl} 2\end{array}$ & Inhibition de Bax & {$[11,12]$} \\
\hline Explants de rétine & Neuroprotection & $\begin{array}{l}\text { Liaison de STI-1 } \\
\text { Activation de PKA }\end{array}$ & {$[13,14]$} \\
\hline \multicolumn{4}{|c|}{ Fonction pro-apoptotique de la PrPc } \\
\hline $\begin{array}{l}\text { Neurones (cervelet } \\
\text { et hippocampe) }\end{array}$ & $\begin{array}{l}\text { Apoptose induite } \\
\text { par pontage avec } \\
\text { des anticorps anti-PrPc }\end{array}$ & $?$ & {$[15]$} \\
\hline $\begin{array}{l}\text { Cellules embryonnaires } \\
\text { de rein (HEK), neurones }\end{array}$ & $\begin{array}{l}\text { Apoptose induite } \\
\text { par la staurosporine }\end{array}$ & $\begin{array}{l}\text { Voie dépendante de p53 } \\
\text { et de la caspase } 3\end{array}$ & {$[16]$} \\
\hline
\end{tabular}

Tableau I. Rôles de la PrPc dans la survie cellulaire.

$$
\text { maintien de l'état d'oxydo-réduction de la cellule [9]. }
$$

\section{Protection contre l'apoptose?}

Des expériences utilisant des neurones en culture provenant de souris $\mathrm{Pr}^{-/-}$semblent indiquer que la $\operatorname{PrP}^{c}$ confère une résistance à l'apoptose induite par une privation de sérum [10]. Dans ces cellules en effet, l'expression de la $\mathrm{Pr} \mathrm{P}^{\mathrm{c}}$, comme celle de la protéine antiapoptotique $\mathrm{Bcl} 2$, identifiée comme possible partenaire d'interaction de la $\mathrm{PrP}^{\mathrm{c}}$, est capable de restaurer une résistance à l'apoptose [11]. Ces données suggèrent ainsi l'implication de la $\mathrm{PrP}^{c}$ dans la régulation de la voie apoptotique dépendante de Bax/Bcl2 qui est prépondérante dans les neurones. À l'appui de cette hypothèse, il a été démontré récemment que la $\operatorname{Pr}^{c}$ empêche les changements de conformation de Bax nécessaires à l'activation de l'apoptose [12]. La fonction anti-apoptotique de la $\operatorname{PrP}^{c}$ est aussi suggérée par l'observation que la protéine STI-1 (stress-inducible protein-1), qui interagit directement avec la $\mathrm{PrP}^{c}$, exerce une activité neuroprotectrice en activant la protéine kinase dépendante de I'AMPc (PKA) [13, 14]. En revanche, des études réalisées sur des coupes tissulaires ont révélé que la liaison de la $\operatorname{Pr} P^{c}$ par des anticorps spécifiques induit l'apoptose des neurones du cervelet et de l'hippocampe [15]. La discordance de ces résultats avec les précédents pourrait être liée à la reconnaissance par les anticorps utilisés d'épitopes distincts.
Certains anticorps utilisés pourraient aussi induire l'activation de la $\operatorname{PrP}^{c}$ en mimant l'interaction avec son ligand physiologique, alors que d'autres au contraire empêcheraient son activation physiologique. D'autres études contredisent également l'hypothèse d'un rôle anti-apoptotique de la $\operatorname{Pr} P^{c}$ : par exemple, la réponse apoptotique à un traitement par la staurosporine (dépendante de p53 et de la caspase 3 ) de cellules embryonnaires de rein (HEK) et de neurones en culture primaire [16] est régulée positivement par l'expression de la PrPc. L'ensemble de ces données (Tableau I) montre qu'il est actuellement impossible de désigner la 
$\mathrm{PrP}^{c}$ comme une protéine pro- ou anti-apoptotique, son implication dans la survie cellulaire dépendant du type cellulaire et de la voie apoptotique impliquée.

\section{Rôle de la PrPc dans l'adhérence cellulaire}

\section{Adhérence cellule-matrice extracellulaire et neuritogenèse}

La $\operatorname{PrP}^{c}$ est une glycoprotéine ancrée à la membrane plasmique par un groupement glycosyl-phophatidylinositol (GPI) au niveau de microdomaines lipidiques, radeaux ou cavéoles (Figure 1 ), où s'accumulent également un grand nombre de protéines impliquées dans la signalisation intracellulaire. Cette localisation suggère pour la $\mathrm{Pr}^{\mathrm{C}}$ une fonction de récepteur et/ou de transmission de signaux intracellulaires et, de fait, plusieurs études ont montré le couplage fonctionnel de la $\operatorname{Pr} P^{c}$ à des voies de signalisation intracellulaires. Ce couplage ne peut pas être direct, la $\operatorname{PrP}^{c}$ ne possédant pas de domaine transmembranaire, et suppose l'interaction avec une protéine transmembranaire. Parmi les partenaires identifiés de la PrPc (Tableau II) figurent plusieurs récepteurs transmembranaires impliqués dans l'adhérence cellulaire: la molécule d'adhérence neuronale NCAM (neural cell adhesion molecule), le précurseur et différentes isoformes du récepteur non-intégrine de la laminine LRP/LR (laminin receptor precursor/laminin receptor) [17-19]. De plus, il est intéressant de noter que la $\operatorname{Pr} P^{c}$ peut également lier directement la laminine et que cette interaction s'est révélée essentielle à la formation de neurites par des neurones d'hippocampe

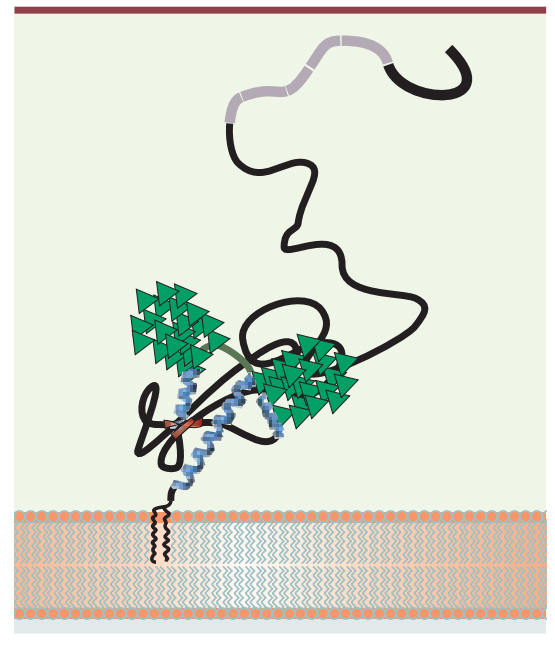

Figure 1. Représentation schématique de la PrPc. La $\mathrm{PrP}^{\mathrm{C}}$ est une glycoprotéine (2 $\mathrm{N}$-glycanes, triangles verts) ancrée à la membrane plasmique par une ancre glycosyl-phosphatidylinositol au niveau de microdomaines de type radeaux lipidiques (dégradé orange). Elle est constituée, dans sa partie C-terminale, de 3 hélices $\alpha$ (bleu) et de 2 petits feuillets $\beta$ (rouge) et dans sa partie amino-terminale, d'une longue chaîne polypeptidique non structurée contenant une région répétée de 5 octapeptides (octarepeat domain, gris) capable de lier les ions $\mathrm{Cu}^{2+}$.

en culture primaire [20]. Inversement, l'inactivation de la $\operatorname{Pr} \mathrm{P}^{c}$ dans la lignée PC12 entraîne une inhibition de la différenciation cellulaire dépendante de la laminine et une rétraction des neurites [21]. Des études plus récentes indiquent que l'interaction de la $\operatorname{Pr} P^{c}$ avec NCAM serait aussi impliquée dans la neuritogenèse : cette réponse implique le recrutement de NCAM par la $\operatorname{PrP}^{c}$ au niveau des radeaux lipidiques et l'activation de la protéine kinase Fyn [22]. STI-1 qui, comme indiqué plus haut, est un partenaire de la $\operatorname{Pr}^{c}$ dont l'activité neuroprotectrice passe par une activation de PKA, pourrait induire la croissance de neurites dans des neurones hippocampiques via l'activation de ERK [23]. L'ensemble de ces données révèle une implication de la $\operatorname{Pr}^{c}$ dans la neuritogenèse, du fait notamment de sa contribution à l'adhérence cellulaire sur une matrice riche en laminine.

\begin{tabular}{|c|c|c|c|}
\hline $\begin{array}{c}\text { Partenaire } \\
\text { d'interaction } \\
\text { (type cellulaire) }\end{array}$ & Fonction cellulaire & $\begin{array}{c}\text { Mécanisme ou } \\
\text { signalisation intracellulaire }\end{array}$ & Réf \\
\hline Laminine (neurone) & $\begin{array}{l}\text { Neuritogenèse en réponse à } \\
\text { NGF sur matrice de laminine }\end{array}$ & Adhérence des cellules à la laminine & {$[19,20]$} \\
\hline LRP/LR (neurone) & $\begin{array}{l}\text { Régulation de l'adhérence } \\
\text { sur matrice de laminine }(s)\end{array}$ & Internalisation de LRP/LR & {$[18,19]$} \\
\hline NCAM (neurone) & Neuritogenèse & $\begin{array}{l}\text { Recrutement de NCAM au niveau } \\
\text { de radeaux lipidiques, } \\
\text { Activation de Fyn }\end{array}$ & {$[17,22]$} \\
\hline STI-1 (neurone) & Neuritogenèse & Activation de $\varepsilon R K$ & [23] \\
\hline $\begin{array}{l}\text { PrPc } \\
\text { (cellule endothéliale) }\end{array}$ & $\begin{array}{l}\text { Adhérence intercellulaire } \\
\text { Migration transendothéliale }\end{array}$ & $\begin{array}{l}\text { Interactions homophiles } \operatorname{Pr}^{\mathrm{c}}-\operatorname{Pr} \mathrm{Pc}^{\mathrm{c}} \\
\text { Interactions homophiles } \operatorname{Pr}^{\mathrm{c}}-\operatorname{Pr} \mathrm{P}^{\mathrm{c}}(s)\end{array}$ & [24] \\
\hline $\begin{array}{l}\operatorname{Pr} P^{c} \\
\text { (cellule épithéliale) }\end{array}$ & Adhérence intercellulaire (s) & Interactions homophiles $\operatorname{Pr}^{c}-\operatorname{Pr} P^{c}(s)$ & [26] \\
\hline
\end{tabular}

Tableau II. Partenaires de la PrPc associés à l'adhérence cellulaire. Dans les cas notés (s), la fonction cellulaire ou le mécanisme associé est seulement suggérée.
Adhérence cellule-cellule et protéine jonctionnelle Une localisation préférentielle de la $\operatorname{PrP}^{c}$ au niveau des jonctions intercellulaires a été récemment observée dans les cellules endothéliales cérébrales qui constituent la barrière hématoencéphalique [24]. Des cultures mixtes de cellules endothéliales cérébrales provenant de souris sauvages ou de souris $\mathrm{PrP}^{-/-}$ont permis d'établir que la localisation jonctionnelle de la $\mathrm{PrPc}^{c}$ est contrôlée par des interactions homophiles [24]. L'interaction PrPc/PrPc entre deux cellules adjacen- 
tes pourrait impliquer une conformation tête-queue similaire à celle qui est décrite pour l'oligomérisation de la PrPc dans un modèle in vitro [25]. Ces observations sont à rapprocher de celles déjà rapportées pour plusieurs molécules d'adhérence jonctionnelles endothéliales comme PECAM-1 (platelet endothelial cell adhesion molecule), VE-cadhérine, ou JAM-A (junctional adhesion molecule). De fait, une colocalisation de la PrPc et de PECAM-1, au sein de microdomaines membranaires jonctionnels (de type radeaux lipidiques), et une analogie fonctionnelle entre ces deux protéines ont été observées dans ces cellules: grâce à l'utilisation d'anticorps spécifiques, il a été montré que la PrPc, comme PECAM-1, est directement impliquée dans la migration de monocytes [24]. Dans des cellules épithéliales, la $\operatorname{PrP}^{c}$ est également exprimée au niveau des jonctions intercellulaires, au sein de microdomaines membranaires du même type, riches en cavéoline-1, où un couplage fonctionnel à la protéine kinase Src pourrait intervenir [26]. L'ensemble de ces observations a permis de proposer que la PrPc pourrait être une protéine jonctionnelle de l'épithélium et de l'endothélium et contrôler des fonctions comme la migration transendothéliale.

\section{Adhérence cellule-cellule et activation lymphocytaire?}

La $\operatorname{PrP}^{c}$ est présente dans les cellules du système immunitaire dont les monocytes, les cellules dendritiques et les lymphocytes. Son expression est augmentée au cours de l'activation des lymphocytes $T$ induite par la concanavaline A ou des anticorps spécifiques de CD3 [27], et la liaison de la $\operatorname{PrP}^{c}$ par des anticorps spécifiques module l'activation lymphocytaire et induit des réarrangements des constituants des radeaux lipidiques et une augmentation de la phosphorylation de protéines de signalisation dont les protéines kinases Src et ERK [28, 29]. Inversement, l'activation cellulaire

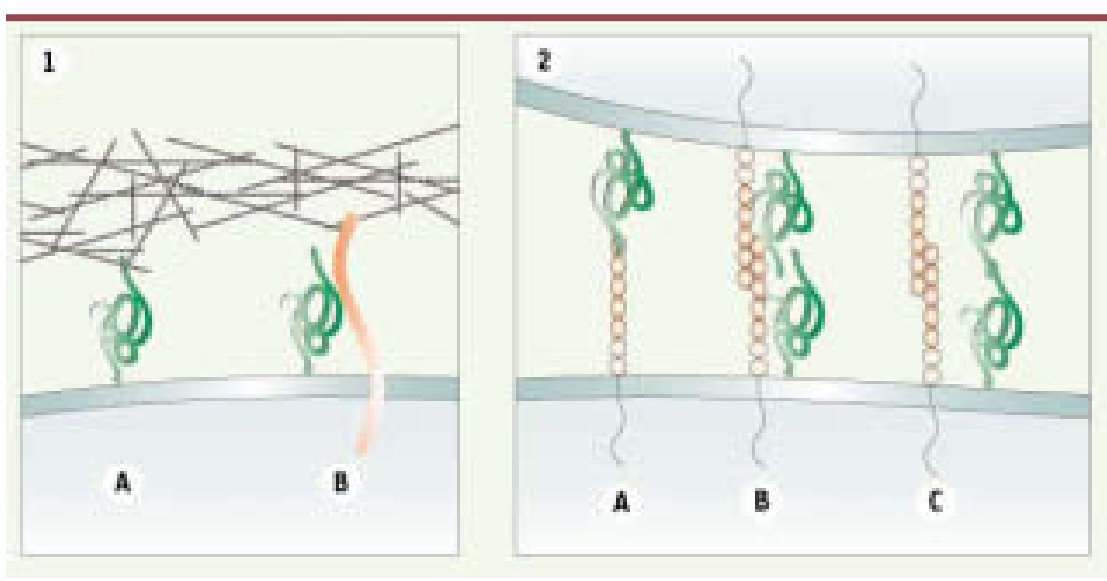

Figure 2. Représentation schématique des interactions impliquant la PrPc dans l'adhérence cellulaire. 1. Adhérence cellule-matrice extracellulaire. La PrPc (vert) peut interagir directement avec des protéines de la matrice extracelullaire (A) comme la laminine ou des récepteurs de protéines de matrice (rouge, $B$ ) comme le précurseur et différentes isoformes du récepteur non-intégrine de la laminine (LRP/LR) et contribuer à l'adhérence cellulaire sur le subtratum. 2. Adhérence cellule-cellule. Au sein de microdomaines lipidiques, la $\operatorname{Pr} P^{c}$ (vert) peut interagir (A, B) ou colocaliser (C) avec des récepteurs trans-membranaires ou des molécules d'adhérence (orange) comme la molécule d'adhérence neuronale NCAM (A, B) ou endothéliale PECAM-1 (C) ; l'interaction avec la molécule d'adhérence pouvant se faire entre deux cellules adjacentes (interaction en trans, $A$ ) ou à la surface d'une même cellule (interaction en cis, B). De manière analogue à la majorité des molécules d'adhérence, la PrPc peut aussi former des interactions homophiles entre deux molécules exprimées sur des cellules adjacentes (C). induite par la concanavaline $A$ ou des anticorps anti-CD3 peut être inhibée en présence d'anticorps bloquant la $\operatorname{Pr}^{c}$ [26]. Bien que les mécanismes moléculaires ne soient pas encore élucidés, il a été observé que la $\operatorname{PrP}^{c}$ est présente au niveau de la synapse immunologique, où elle colocalise avec le récepteur à l'antigène des cellules T (TCR) notamment lorsque celui-ci se lie à des anticorps spécifiques. À la surface des cellules dendritiques, la PrPc est aussi colocalisée avec les molécules du complexe majeur d'histocompatibilité de classe II, qui, après leur liaison à l'antigène, interagissent avec le TCR des lymphocytes T. Enfin, l'étude de l'interaction entre lymphocytes $T$ et cellules dendritiques exprimant ou non la $\operatorname{Pr}^{\mathrm{C}}$ a permis de suggérer fortement l'implication de la PrPc dans cette interaction et dans l'activation lymphocytaire [30].

\section{Conclusions}

Quelles sont donc les fonctions de la PrPc ? Plusieurs approches ont été mises en œuvre et ont abouti à l'identification de multiples partenaires potentiels de la $\operatorname{Pr}^{c}$ dont la signification physiologique reste, pour la majorité d'entre eux, à démontrer. L'absence de phénotype majeur des souris $\mathrm{Pr}^{-/-}$, dans des conditions normales de développement et de vie, a aussi conduit à considérer la $\mathrm{PrP}^{\mathrm{c}}$ comme une protéine «non essentielle». Cependant, des études in vivo ont révélé une contribution possible de la $\mathrm{Pr} \mathrm{P}^{\mathrm{c}}$ à plusieurs réponses comportementales, ces réponses étant de fait perturbées dans les maladies à prion. On peut citer par exemple la régulation du sommeil, l'apprentissage et la persistance de la mémoire. Bien que les mécanismes moléculaires responsables de ces réponses restent largement incompris, des résultats in vitro récents ont permis de mettre en évidence deux paramètres biologiques dans lesquels la $\operatorname{Pr}^{c}$ semble impliquée: la survie cellulaire et l'adhérence cellulaire. En effet, il est intéressant de souligner le rôle majeur de l'adhérence cellulaire dans les fonctions régulées par la $\operatorname{Pr} \mathrm{P}^{\mathrm{c}}$, même si ces dernières varient sensiblement selon le type cellulaire. Dans les neurones, cellules les plus étudiées à ce jour, la $\operatorname{Pr} P^{c}$, par sa capacité de réguler l'adhérence cellulaire, contribue à la fois à la neuritogenèse et à la survie cellulaire. Dans les cellules épithéliales et endothéliales, toutes deux constituant des barrières physiologiques, la $\mathrm{PrP}^{\mathrm{c}}$ est localisée au niveau des jonctions inter-cellulaires et apparaît capable de réguler des fonctions 
généralement associées aux molécules d'adhérence, notamment la migration de monocytes à travers l'endothélium. Finalement, il est à noter que la contribution de la PrPc à l'activation des lymphocytes T pourrait aussi être liée à sa capacité de stabilisation des contacts inter-cellulaires entre lymphocytes $T$ et cellules présentatrices d'antigène, au niveau de la synapse immunologique. Ces observations suggèrent ainsi que la $\operatorname{Pr} \mathrm{P}^{c}$ pourrait avoir un rôle clé de stabilisateur de l'adhérence cellulaire en interagissant avec des protéines de la matrice extracellulaire, des récepteurs impliqués dans l'adhérence cellulaire et/ou avec la $\mathrm{PrP}^{\mathrm{C}}$ exprimée par la cellule adjacente (Figure 2). Il apparaît également, au vu de ces données, que les fonctions de la $\operatorname{Pr} \mathrm{PC}^{\mathrm{c}}$ pourraient se révéler particulièrement importantes dans des situations pathologiques comme lors d'un stress cellulaire, de pathologies inflammatoires ou infectieuses. II est maintenant envisageable que les données récentes concernant les fonctions normales de la $\operatorname{PrP}^{c}$ puissent conduire, en améliorant notre compréhension des mécanismes moléculaires responsables des maladies à prion, à l'élaboration de stratégies thérapeutiques pour ces maladies. $\diamond$

\section{SUMMARY}

\section{Functions of prion protein PrPc}

It is now well established that both normal and pathological (or scrapie) isoforms of prion protein, $\mathrm{PrP}^{\mathrm{c}}$ and $\mathrm{Pr} \mathrm{P}^{\mathrm{sc}}$ respectively, are involved in the development and progression of various forms of neurodegenerative diseases, including scrapie in sheep, bovine spongiform encephalopathy (or «mad cow disease ») and Creutzfeldt-Jakob disease in human, collectively known as prion diseases. The protein $\mathrm{Pr}^{\mathrm{c}}$ is highly expressed in the central nervous system in neurons and glial cells, and also present in non-brain cells, such as immune cells or epithelial and endothelial cells. Identification of the physiological functions of $\mathrm{Pr} \mathrm{P}^{\mathrm{C}}$ in these different cell types thus appears crucial for understanding the progression of prion diseases. Recent studies highlighted several major roles for $\operatorname{PrP}^{c}$ that may be considered in two major domains : (1) cell survival (protection against oxidative stress and apoptosis) and (2) cell adhesion. In association with cell adhesion, distinct functions of $\mathrm{Pr}^{\mathrm{c}}$ were observed, depending on cell types: neuronal differentiation, epithelial and endothelial barrier integrity, transendothelial migration of monocytes, $T$ cell activation. These observations suggest that $\mathrm{PrP}^{\mathrm{c}}$ functions may be particularly relevant to cellular stress, as well as inflammatory or infectious situations. $\diamond$

\section{REMERCIEMENTS}

Cette étude a bénéficié du soutien financier du programme «GIS Infection à prion », de l'Institut National pour la Santé et la Recherche Médicale (INSERM), du Centre National de la Recherche Scientifique (CNRS), de l'Université Paris V, du Ministère de la Jeunesse, de l'Éducation nationale et de la Recherche et de la Fundaçao para a Ciencia e Tecnologia du Portugal.

\section{RÉFÉRENCES}

1. Prusiner SB. Novel proteinaceous infectious particles cause scrapie. Science $1982 ; 216: 136-44$.

2. Brown DR, Qin K, Herms JW, et al. The cellular prion protein binds copper in vivo. Nature 1997 ; $390: 684-7$.

3. Brown DR, Besinger A. Prion protein expression and superoxide dismutase activity. Biochem J 1998 ; $334: 423-9$.

4. Gaeta A, Hider RC. The crucial role of metal ions in neurodegeneration : the basis for a promising therapeutic strategy. Br J Pharmacol 2005 ; 146 : 1041-59.

5. Dupuis L, Loeffler JP. Protéine cellulaire du prion : au-delà des encéphalopathies spongiformes. Med Sci (Paris) $2003 ; 19: 783-5$

6. Brown DR, Schulz-Schaeffer WJ, Schmidt B, Kretzschmar HA. Prion protein-deficient cells show altered response to oxidative stress due to decreased SOD-1 activity. Exp Neurol 1997 ; 146 : 104-12.

7. Schneider B, Mutel V, Pietri M, et al. NADPH oxidase and extracellular regulated kinases $1 / 2$ are targets of prion protein signaling in neuronal and nonneuronal cells. Proc Natl Acad Sci USA 2003; $100: 13326-31$.

8. Mouillet-Richard S, Ermonval M, Chebassier C, et al. Signal transduction through prion protein. Science $2000 ; 289$ : 1925-8.

9. Schneider B, Pietri M, Ermonval M, et al. Contrôler l'équilibre redox cellulaire : une fonction ubiquitaire pour la protéine prion cellulaire? Med Sci (Paris) $2004 ; 20: 21-3$.

10. Kim BH, Lee HG, Choi JK, et al. The cellular prion protein (PrPC) prevents apoptotic neuronal cell death and mitochondrial dysfunction induced by serum deprivation. Brain Res Mol Brain Res 2004 ; $124: 40-50$.

11. Bounhar $Y$, Zhang $Y$, Goodyer CG, LeBlanc A. Prion protein protects human neurons against Baxmediated apoptosis. J Biol Chem $2001 ; 276$ : 39145-9.

12. Saupe SJ, Supattapone S. What makes a good prion? Conference on Prion Biology. EMBO Rep 2006 ; $7: 254-8$.

13. Zanata SM, Lopes MH, Mercadante AF, et al. Stress-inducible protein 1 is a cell surface ligand for cellular prion that triggers neuroprotection. EMBO J $2002 ; 21: 3307-16$.

14. Chiarini LB, Freitas AR, Zanata SM, et al. Cellular prion protein transduces neuroprotective signals. EMBO J $2002 ; 21: 3317-26$.

15. Solforosi L, Criado JR, McGavern DB, et al. Cross-linking cellular prion protein triggers neuronal apoptosis in vivo. Science $2004 ; 303$ : 1514-6.

16. Paitel $\varepsilon$, Sunyach C, Alves da Costa C, et al. Primary cultured neurons devoid of cellular prion display lower responsiveness to staurosporine through the control of $p 53$ at both transcriptional and post-transcriptional levels. J Biol Chem $2004 ; 279: 612-8$.

17. Schmitt-Ulms G, Legname G, Baldwin MA, et al. Binding of neural cell adhesion molecules ( $N$-CAMs) to the cellular prion protein. J Mol Biol $2001 ; 314: 1209-25$.

18. Rieger $\mathrm{R}$, Edenhofer $\mathrm{F}$, Lasmezas $\mathrm{Cl}$, Weiss $\mathrm{S}$. The human $37-\mathrm{kDa}$ laminin receptor precursor interacts with the prion protein in eukaryotic cells. Nat Med $1997 ; 3: 1383-8$.

19. Simoneau $S$, Haik S, Leucht $C$, et al. Different isoforms of the non-integrin laminin receptor are present in mouse brain and bind PrP. J Biol Chem 2003; $384: 243-6$.

20. Graner $\varepsilon$, Mercadante AF, Zanata SM, et al. Cellular prion protein binds laminin and mediates neuritogenesis. Brain Res Mol Brain Res $2000 ; 76: 85-92$.

21. Graner $E$, Mercadante AF, Zanata SM, et al. Laminin-induced PC-12 cell differentiation is inhibited following laser inactivation of cellular prion protein. FEBS Lett $2000 ; 482: 257-60$.

22. Santuccione A, Sytnyk V, Leshchyns'ka I, Schachner M. Prion protein recruits its neuronal receptor NCAM to lipid rafts to activate p59fyn and to enhance neurite outgrowth. J Cell Biol 2005; 169: 341-54.

23. Lopes MH, Hajj GN, Muras AG, et al. Interaction of cellular prion and stress-inducible protein 1 promotes neuritogenesis and neuroprotection by distinct signaling pathways. J Neurosci 2005 ; $25: 11330-9$.

24. Viegas $P$, Chaverot $N$, Enslen $H$, et al. Junctional expression of the prion protein PrPc by brain endothelial cells : a role in trans-endothelial migration of human monocytes. J Cell Sci 2006 ; $119: 4634-43$.

25. Knaus KJ, Morillas M, Swietnicki W, et al. Crystal structure of the human prion protein reveals a mechanism for oligomerization. Nat Struct Biol $2001 ; 8: 770-4$.

26. Morel $\varepsilon$, Fouquet $S$, Chateau $D$, et al. The cellular prion protein $\mathrm{PrPc}$ is expressed in human enterocytes in cell-cell junctional domains. J Biol Chem $2004 ; 279: 1499-505$.

27. Li R, Liu D, Zanusso G, et al. The expression and potential function of cellular prion protein in human lymphocytes. Cell Immunol $2001 ; 207: 49-58$.

28. Hugel B, Martinez MC, Kunzelmann C, et al. Modulation of signal transduction through the cellular prion protein is linked to its incorporation in lipid rafts. Cell Life Mol Sci 2004 ; 61 : 2998-3007.

29. Stuermer CA, Langhorst MF, Wiechers MF, et al. PrPc capping in T cells promotes its association with the lipid raft proteins reggie-1 and reggie-2 and leads to signal transduction. FASEB J 2004 ; $18: 1731-3$.

30. Ballerini C, Gourdain P, Bachy V, et al. Functional implication of cellular prion protein in antigendriven interactions between T cells and dendritic cells. J Immunol 2006 ; 176 : 7254-62. 